(C) 2007 IEEE. Personal use of this material is permitted. Permission from IEEE must be obtained for all other uses, in any current or future media, including reprinting/republishing this material for advertising or promotional purposes, creating new collective works, for resale or redistribution to servers or lists, or reuse of any copyrighted component of this work in other works. 


\title{
Z-Transform-Based FDTD Analysis of Perfectly Conducting Cylinder Covered with Unmagnetized Plasma
}

\author{
Yan Ming, Shao K.R., Hu X.W., Guo Youguang, Zhu Jianguo, Lavers J.D., Fellow IEEE
}

\begin{abstract}
In this paper a novel and normalized Z-Transformbased finite-difference time-domain (ZTFDTD) method is presented for simulating the interaction of electromagnetic (EM) wave with unmagnetized plasma. The 2-D ZTFDTD formulations for unmagnetized plasma are derived. Using a simplified 2-D model for perfectly conducting cylinder covered with unmagnetized plasma, the stealth effect of unmagnetized plasma is studied in different thicknesses of plasma, electron densities of plasma, EM wave frequencies and plasma collision frequencies. Numerical results indicate that plasma stealth is effective in theory and the reasonable selection for the plasma parameters can greatly enhance its effectiveness.
\end{abstract}

Index Terms-FDTD, plasma stealth, radar cross section (RCS) , Z-Transform.

\section{INTRODUCTION}

$\mathrm{T}$ he plasma stealth is a novel technology in theory. The study of target plasma stealth has attracted much attention from the United States, Russia and other countries around the world because of its advantages. A lot of work has been previously done in plasma stealth and modeling plasma with the FDTD technique. Vidmar [1] discussed that the absorber of EM wave depends on incident wave frequency, plasma density, and momentum-transfer collision rate. Laroussi [2] studied numerical results of the reflection, absorption, and transmission of microwaves by a magnetized nonuniform plasma slab. Shi [3] discussed the problems of electromagnetic reflection of conductive plane covered with magnetized inhomogeneous plasma by using numerical method, where the plasma electron density profile is assumed as parabolic, and the external magnetic field also varies across the plasma. Nickisch [4] integrated the dynamical equations of dielectric tensor with Maxwell's equations and implemented

Manuscript received October 31, 2006. This work was supported by NSFC under Grant No. 90405004

Ming Yan, K.R. Shao and X.W. Hu are with the College of Electrical and Electronic Engineering, Huazhong University of Sci. \& Tech.,Wuhan, China. (phone:+86-27-87542626, e-mail: krshao@hust.edu.cn)

Youguang Guo and Jianguo Zhu are with the faculty of Engineering, University of Technology, Sydney, NSW, Australia. (e-mail: Youguang.Guo (a)eng.sut.edu.au).

J.D. Lavers is with the Dept. of E \& C Engineering, University of Toronto, Toronto, ON, M5S 3G4 Canada. (e-mail: lavers@ecf.utoonto.ca) an FDTD model. Luebber [5] considered the dispersive plasma in addition to Debye dielectrics. However, the functional form of the formulation made use diverges as the electron collision frequency goes to zero, and hence it is restricted to plasma exhibiting significant absorption. They described an FDTD formulation which incorporates both anisotropy and frequency dispersion at the same time enabling the transient analysis of magneto active plasma.

In this paper a novel and normalized Z-Transform-based finite-difference time- domain (ZTFDTD) method is presented for simulating the interaction of EM wave with unmagnetized plasma. This FDTD method is originally suggested by Sullivan [6]-[7] and has been applied to dispersive media. The main purpose of this paper is to induce the ZTFDTD method into plasma stealth technology. The 2-D ZTFDTD formulations for unmagnetized plasma are derived. Using a simplified 2-D model for perfectly conducting cylinder covered with unmagnetized plasma, the stealth effect of unmagnetized plasma is studied in different thicknesses of plasma, electron densities of plasma, EM wave frequencies and plasma collision frequencies.

\section{Z-TRANSFORM-BASED FINITE-DIFFERENCE TIME-DOMAIN}

A more general form of Maxwell's equations which uses the $E, H, D$ fields is

$$
\begin{aligned}
& \frac{\partial \boldsymbol{D}}{\partial \boldsymbol{t}}=\nabla \times \boldsymbol{H} \\
& \boldsymbol{D}(\omega)=\varepsilon_{0} \cdot \varepsilon_{r}^{*}(\omega) \cdot \boldsymbol{E}(\omega) \\
& \frac{\partial \boldsymbol{H}}{\partial \boldsymbol{t}}=-\frac{1}{\mu_{0}} \nabla \times \boldsymbol{E}
\end{aligned}
$$

where $D$ is the electric flux density, $E$ is the electric field, $H$ is the magnetic intensity. Because $\mu$ and $\varepsilon$ differ by several orders of magnitude, the $E$ field and $H$ field will differ by several orders of magnitude. This can be avoided by making the following change of variables:

$$
\tilde{\boldsymbol{E}}=\sqrt{\frac{\varepsilon_{0}}{\mu_{0}}} \cdot \boldsymbol{E}, \tilde{\boldsymbol{D}}=\sqrt{\frac{1}{\varepsilon_{0} \cdot \mu_{0}}} \cdot \boldsymbol{D}
$$

Substituting (4) into (1), (2), and (3) gives

$$
\frac{\partial \tilde{\mathbf{D}}}{\partial \boldsymbol{t}}=\sqrt{\frac{1}{\varepsilon_{0} \cdot \mu_{0}}} \nabla \times \boldsymbol{H}
$$




$$
\begin{aligned}
& \tilde{\boldsymbol{D}}(\omega)=\varepsilon_{\boldsymbol{r}}^{*}(\omega) \cdot \tilde{\boldsymbol{E}}(\omega) \\
& \frac{\partial \boldsymbol{H}}{\partial \boldsymbol{t}}=-\sqrt{\frac{1}{\varepsilon_{0} \cdot \mu_{0}}} \nabla \times \tilde{\boldsymbol{E}}
\end{aligned}
$$

Equations (5) and (7) will lead to very simple finite difference equations. The only change is to use variable $D$ instead of E. Equation (6) has to be formulated into a time domain difference equation for implementation of FDTD. The first task is to get it from the frequency domain to time domain. Using the Wentzel- Kramers-Brillouin (WKB) approximation [8], the permittivity of unmagnetized plasma is described as follows:

$$
\begin{aligned}
& \varepsilon_{r}^{*}(\omega)=1+\frac{\omega_{p}^{2}}{\omega\left(j v_{c}-\omega\right)} \\
& \omega_{p}=2 \pi f_{p}=\left(N_{e} e^{2} / m_{e} \varepsilon_{0}\right)^{1 / 2}
\end{aligned}
$$

where $f_{p}$ is the plasma frequency, $v_{c}$ is the electron collision frequency, $N_{e}$ is the electron density, $e$ is the electron charge and $m_{e}$ is the mass of the electron.

In frequency-dependent media, we can avoid dealing with troublesome convolution integrals in the time domain using $\mathrm{Z}$ transforms for the FDTD formulation. By using partial fraction expansion and $\mathrm{Z}$ transform, (8) can be written as

$$
\varepsilon_{r}^{*}(z)=\frac{1}{\Delta t}+\frac{\omega_{p}^{2} / v_{c}}{1-z^{-1}}-\frac{\omega_{p}^{2} / \nu_{c}}{1-e^{-v_{c} \cdot \Delta t} z^{-1}}
$$

By the convolution theorem, the $\mathrm{Z}$ transform of (6) is

$$
\tilde{D}(z)=\Delta t \cdot \varepsilon_{r}^{*}(z) \cdot \tilde{E}(z)
$$

By inserting (10) into (11), we obtain

$$
\tilde{D}(z)=\tilde{E}(z)+\frac{\omega_{p}^{2} \Delta t}{v_{c}}\left[\frac{\left(1-e^{-v_{c} \cdot \Delta t}\right) z^{-1}}{1-\left(1-e^{-v_{c} \cdot \Delta t}\right) z^{-1}+e^{-v_{c} \cdot \Delta t} z^{-2}}\right] \tilde{E}(z)
$$

An auxiliary term will be defined as

$$
M(z)=\frac{\omega_{p}^{2} \Delta t}{v_{c}}\left[\frac{\left(1-e^{-v_{c} \cdot \Delta t}\right)}{1-\left(1-e^{-v_{c} \cdot \Delta t}\right) z^{-1}+e^{-v_{c} \cdot \Delta t} z^{-2}}\right] \tilde{E}(z)
$$

$E(z)$ can be solved for by

$$
\tilde{E}(z)=D(z)-z^{-1} M(z)
$$

Therefore, the FDTD simulation becomes

$$
\begin{aligned}
\tilde{E}^{n}= & \tilde{D}^{n}-M^{n-1} \\
M^{n} & =\left(1+e^{-v_{c} \cdot \Delta t}\right) M^{n-1}-e^{-v_{c} \cdot \Delta t} M^{n-2} \\
& +\frac{\omega_{p}^{2} \Delta t}{v_{c}}\left(1-e^{-v_{c} \cdot \Delta t}\right) \tilde{E}^{n}
\end{aligned}
$$

\section{ZTFDTD ANALYSIS OF PERFECTLy CONDUCTING CYLINDER COVERED WITH UNMAGNETIZED PlasMA}

The plasma can be satisfied in a wide range of frequency bands with a strong absorption capacity of the incident wave, while reducing the reflection of the incident EM wave. In order to reduce the radar cross section (RCS), it is necessary to choose the distribution function of plasma electron density reasonably. Ideal plasma boundary condition is

$$
\left\{\begin{array}{l}
N_{e}\left(r_{2}\right)=0, n\left(r_{2}\right)=1 \\
N_{e}\left(r_{1}\right)=N_{e 0}, n\left(r_{1}\right)=0
\end{array}\right.
$$

where $N_{e}$ is the electron density, $n$ is the refractive index, $N_{e o}$ is the peak value electron density. According to (16), the electron density of plasma is taken as:

$$
N_{e}(x)=N_{e 0} \cdot\left(r_{2}-r\right)^{2 m} /\left(r_{2}-r_{1}\right)^{2 m}
$$

Substituting (17) into (9):

$$
\omega_{p}(r)=\omega_{p \max } \cdot\left(r_{2}-r\right)^{m} /\left(r_{2}-r_{1}\right)^{m}
$$

To demonstrate the aforementioned ZTFDTD formulation for unmagnetized plasma, we compute the RCS of EM wave through a perfectly conducting cylinder covered with unmagnetized plasma. Fig.1 shows three boundaries and zoning of the FDTD grid. The incident wave is imported to equations (5)-(7) by connecting boundary, and the reflected wave of EM wave is absorbed by UPML. Using a simplified 2-D model-perfectly conducting cylinder (The radius is $10 \mathrm{~cm}$ ) covered with unmagnetized plasma, the stealth effect of unmagnetized plasma is studied in different thicknesses of plasma, electron densities of plasma, EM wave frequencies and plasma collision frequencies. The incident EM wave is sinusoidal plane wave $\left(E_{i n}=\sin (2 \pi f t)\right)$

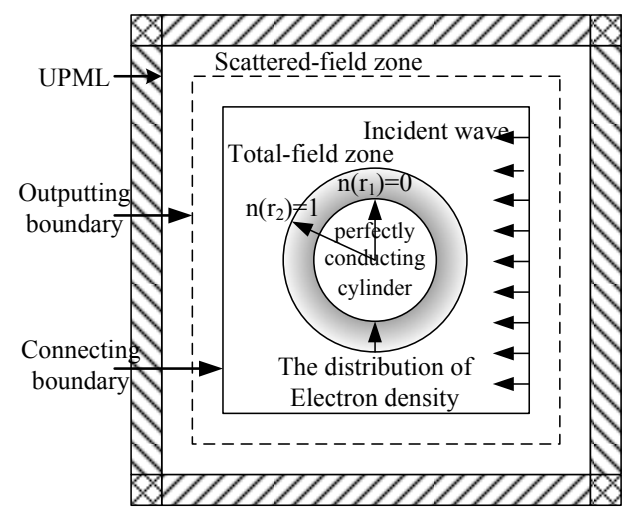

Fig. 1 boundaries and zoning of the fdtd grid

Fig. 2 shows RCS of perfectly conducting cylinder covered with nothing (ZTFDTD and analytical solution [9]), homogeneous and inhomogeneous unmagnetized plasma (HUP and IUP). The electron density, plasma frequency, plasma collision frequency of HUP are, respectively, $1.12 \times 10^{17} / \mathrm{m}^{3}, 3 \mathrm{GHz}$ and $10 \mathrm{GHz}$. the value of $\mathrm{m}$, maximum electron density, plasma frequency, plasma collision frequency of IUP are, respectively, $1,1.12 \times 10^{17} / \mathrm{m}^{3}, 3 \mathrm{GHz}$ and $10 \mathrm{GHz}$. The EM wave frequency is $3 \mathrm{GHz}$. The HUP and IUP all have the absorption to the incident EM wave, but the IUP is better. This is mainly due to the fact that the edge of the HUP obviously is not continuous, and it can create the stronger reflection for incident EM wave, while the IUP has a better continuity.

Fig. 3 shows RCS of perfectly conducting cylinder covered with IUP in different electron densities $(\mathrm{m}=0.5,1,1.5,2)$. The EM wave frequency is $3 \mathrm{GHz}$. The plasma collision frequency, maximum electron density and corresponding plasma frequency of HUP are, respectively, $20 \mathrm{GHz}, 1.12 \times 10^{17} / \mathrm{m}^{3}$ and $3 \mathrm{GHz}$. In this condition the plasma stealth is effective. The smaller the value of $\mathrm{m}$ is, the more remarkable the plasma stealth effect is. 


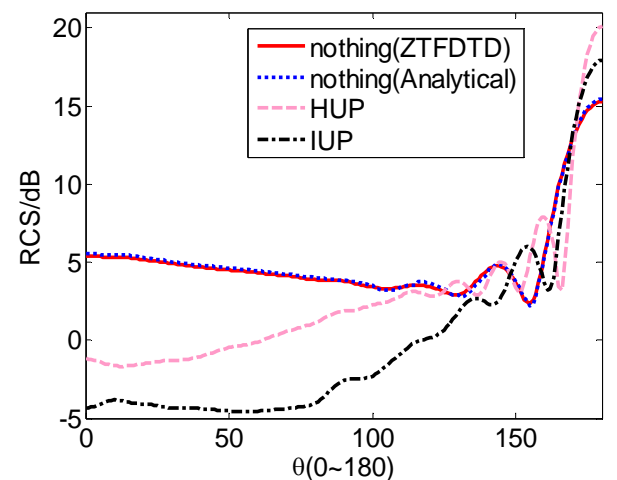

Fig.2 RCS of perfectly conducting cylinder covered with nothing, HUP and IUP

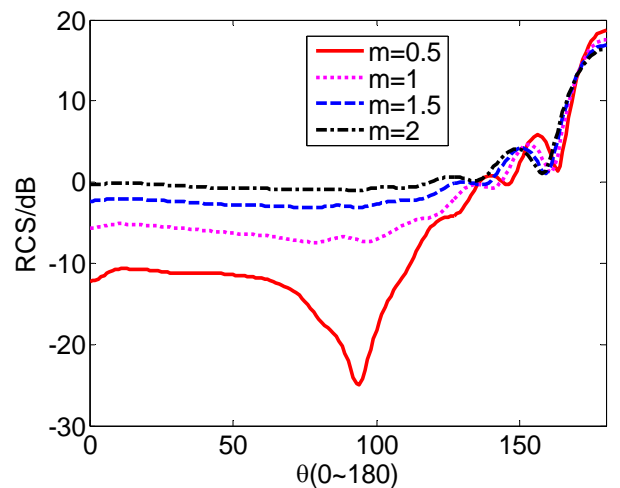

Fig.3 RCS of perfectly conducting cylinder covered with IUP in different electron densities

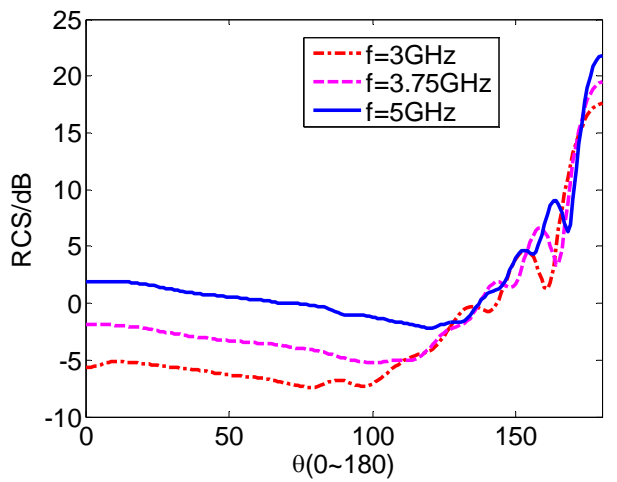

Fig.4 RCS of perfectly conducting cylinder covered with IUP in different EM wave frequencies

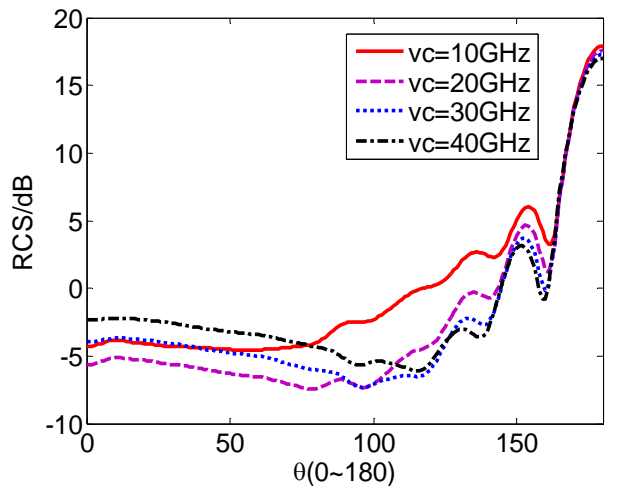

Fig.5 RCS of perfectly conducting cylinder covered with IUP in different plasma collision frequencies
Fig.4 shows RCS of perfectly conducting cylinder covered with IUP in different EM wave frequencies $(\mathrm{f}=3,3.75,5 \mathrm{GHz})$. The value of $\mathrm{m}$, plasma collision frequency, maximum electron density and corresponding plasma frequency of HUP are, respectively, $1,20 \mathrm{GHz}, 1.12 \times 10^{17} / \mathrm{m}^{3}$ and $3 \mathrm{GHz}$. The ability of IUP absorption different frequency EM wave is much different. When the EM wave frequency approaches to the lower limit of plasma frequency, the attenuation of EM wave is increased because of the plasma resonance absorption of EM waves.

Fig. 5 shows RCS of perfectly conducting cylinder covered with IUP in different plasma collision frequencies $\left(v_{c}=10,20\right.$, $30,40 \mathrm{GHz}$ ). The EM wave frequency is $3 \mathrm{GHz}$. The value of $\mathrm{m}$, maximum electron density and corresponding plasma frequency of HUP are, respectively, $1,1.12 \times 10^{17} / \mathrm{m}^{3}$ and $3 \mathrm{GHz}$. The plasma collision frequency has a best value. When the plasma has this collision frequency, the attenuation of EM wave is biggest.

From Fig.2 to Fig.5 we can find that when the bistatic angle of the radar system is zero or small, the plasma can enormously reduce the RCS. Alternatively, when the bistatic angle is large, the plasma increases RCS.

\section{CONCLUSION}

A novel ZTFDTD method is applied to study the RCS of perfectly conducting cylinder covered with unmagnetized plasma, Numerical results indicate that plasma stealth is effective in theory and reasonable selection of the plasma parameters can greatly enhance its effectiveness.

\section{REFERENCES}

[1] R.J. Vidmer. "On the use of atmospheric pressure plasma as electromagnetic reflectors and absorbers," IEEE Trans. Plasma Sci., vol. 18, pp. 733-741, Apr.1990.

[2] M. Laroussi and J.R. Roth, "Numerical calculation of the reflection, absorption, and transmission of microwaves by a nonuniform plasma slab," IEEE Trans. Plasma Sci., vol. 2, pp. 366-371, Aug.1993.

[3] J.M. Shi, "Electromagnetic reflection of conductive plane covered with magnetized inhomogeneous plasma," International Journal of Infrared and Millimeter Waves, vol. 22, pp. 1167-1175, May.1990.

[4] L.J. Nickisch and P.M. Franke, "Finite-difference time-domain solution of Maxwell's equations for the dispersive ionosphere," IEEE Trans. Antennas Propagat., vol. 34, pp. 33-39, May.1990.

[5] R.J. Luebbers, F. Hunsberger, K.S. Kunz, "A frequency-dependent finite difference time-domain formulation for transient propagation in plasma," IEEE Trans. Antennas Propagat., vol. 39, pp. 29-34, Jan.1991.

[6] D.M. Sullivan, "Electromagnetic simulation using the FDTD method," New York: IEEE Press, 2000.

[7] D.M. Sullivan, "Frequency-dependent FDTD method using Z transforms," IEEE Trans. Antennas Propagat., vol. 40, pp. 1223-1230, Oct. 1992.

[8] Shu Zhang, Xiwei $\mathrm{Hu}$, Zhonghe Jiang, "Propagation of an electromagnetic wave in an atmospheric pressure plasma: Numerical solutions," Physics of Plasma, vol. 13, pp. 1-7, 2006.

[9] C. Balanis, "Advanced Engineering Electromagnetics," New York: John Wiley \& Sons, 1989. 